\title{
ON THE TRANSIT POTENTIAL OF THE PLANET ORBITING IOTA DRACONIS
}

\author{
Stephen R. Kane ${ }^{1}$, Sabine Reffert $^{2}$, Gregory W. Henry ${ }^{3}$, Debra Fischer ${ }^{4}$, Christian Schwab $^{2}$, Kelsey I. Clubi $^{4}$, \\ AND CHRISTOPH BERGMANN ${ }^{2}$ \\ ${ }^{1}$ NASA Exoplanet Science Institute, California Institute of Technology, MS 100-22, 770 South Wilson Avenue, Pasadena, CA 91125, USA; skane@ipac.caltech.edu \\ ${ }^{2}$ ZAH-Landessternwarte, Königstuhl 12, 69117 Heidelberg, Germany \\ ${ }^{3}$ Center of Excellence in Information Systems, Tennessee State University, 3500 John A. Merritt Blvd., Box 9501, Nashville, TN 37209, USA \\ ${ }^{4}$ Department of Physics \& Astronomy, San Francisco State University, San Francisco, CA 94132, USA \\ Received 2010 May 17; accepted 2010 July 16; published 2010 August 25
}

\begin{abstract}
Most of the known transiting exoplanets are in short-period orbits, largely due to the bias inherent in detecting planets through the transit technique. However, the eccentricity distribution of the known radial velocity planets results in many of those planets having a non-negligible transit probability. One such case is the massive planet orbiting the giant star iota Draconis, a situation where both the orientation of the planet's eccentric orbit and the size of the host star inflate the transit probability to a much higher value than for a typical hot Jupiter. Here we present a revised fit of the radial velocity data with new measurements and a photometric analysis of the stellar variability. We provide a revised transit probability, an improved transit ephemeris, and discuss the prospects for observing a transit of this planet from both ground and space.
\end{abstract}

Key words: planetary systems - stars: individual ( Draconis) - techniques: photometric

\section{INTRODUCTION}

The detection of exoplanetary transits has revolutionized the way we view giant planets, both in their variety of structures (Batygin et al. 2009) and their formation processes (Veras et al. 2009). Even so, this view is mostly restricted to planets in short-period circular orbits around their host stars. The groundbased transit detection of the planets HD 17156b (Barbieri et al. 2007) and HD 80606b (Laughlin et al. 2009; Moutou et al. 2009), enabled by their high eccentricities (Kane \& von Braun 2008, 2009), provided the first insights into the structures of longer-period planets. Further discoveries of long-period planetary transits around bright stars are vital to understanding the dependence of planetary structure/evolution on the periastron distance of the planet (Kane et al. 2009). Provided the orbital parameters can be determined with sufficient precision, monitoring planets detected via the radial velocity technique at predicted transit times provides a means to increase the sample of long-period transiting planets. Efforts to detect transits of the known radial velocity planets are currently being undertaken by the Transit Ephemeris Refinement and Monitoring Survey (TERMS).

Amongst the radial velocity planet discoveries, a substantial number have been found to orbit giant stars. Recent examples of such discoveries include the planetary companions of 11 Ursae Minoris and HD 32518 (Döllinger et al. 2009) and HD 102272 (Niedzielski et al. 2009). These detections are in good agreement with predictions of their frequency and dependence on the host star mass (Ida \& Lin 2005; Kennedy \& Kenyon 2008). In particular, planets orbiting giant stars tend to have large transit probabilities due to the size of the host stars (Assef et al. 2009). Giant stars present significant challenges, however, to those who intend to monitor those stars for the purpose of detecting exoplanetary transits. A good example is the planet orbiting HD 122430 (Setiawan et al. 2004), for which the combination of the $22.9 R_{\odot}$ host star (da Silva et al. 2006) and the high eccentricity of the planetary orbit lead to a transit probability of $\sim 32 \%$. However, assuming a Jupiter radius for the planet requires the unambiguous detection of a $1.9 \times 10^{-5}$ transit depth. Additionally, the uncertainty in the orbital parameters of the planet means that a large transit window will need to be continuously monitored (Kane et al. 2009). Finally, a photometric survey of giant stars by Henry et al. (2000) found that almost half of their sample exhibited low-level photometric variability that would further complicate transit detection.

The planet orbiting iota Draconis (hereafter $\iota$ Dra) presents a particularly interesting case. The host star is a K2 giant, is very bright $(V=3.29)$, and is frequently referred to by its other common aliases of HD 137759 and HIP 75458. A thorough spectral analysis of this star was undertaken by Sadakane et al. (2005), who found a metallicity of $[\mathrm{Fe} / \mathrm{H}]=0.12$. The planetary companion was discovered by Frink et al. (2002), and the orbit was further refined by Zechmeister et al. (2008), whose radial velocity data revealed a linear trend over time. In addition to the large stellar radius, the planetary orbit is highly eccentric and the argument of periastron $\left(\omega \sim 90^{\circ}\right)$ ensures that the periastron passage occurs approximately in the observer-star plane perpendicular to the line of sight.

Here we present new radial velocity data for $\iota$ Dra $b$ and an analysis of the photometric stability of the host star. These data are used to provide a well-constrained transit ephemeris for the next 10 years and an assessment of the feasibility of detecting a transit for the planetary companion. This analysis and discussion may be used as a model for how to consider the transit detection potential for each of the planets orbiting giant stars. In Section 2, we present the new radial velocity data and revised orbital solution. Section 3 discusses the geometric transit probability of the planet and the predicted transit depth, and in Section 4 we calculate the transit ephemeris for the planet. Section 5 presents the photometric analysis of the host star, and in Section 6 we discuss the potential for detecting a transit of the planet.

\section{ORBITAL SOLUTION}

The initial orbital solution provided by Frink et al. (2002) had relatively large uncertainties associated with the orbital parameters because they had only a single cycle of the planetary 

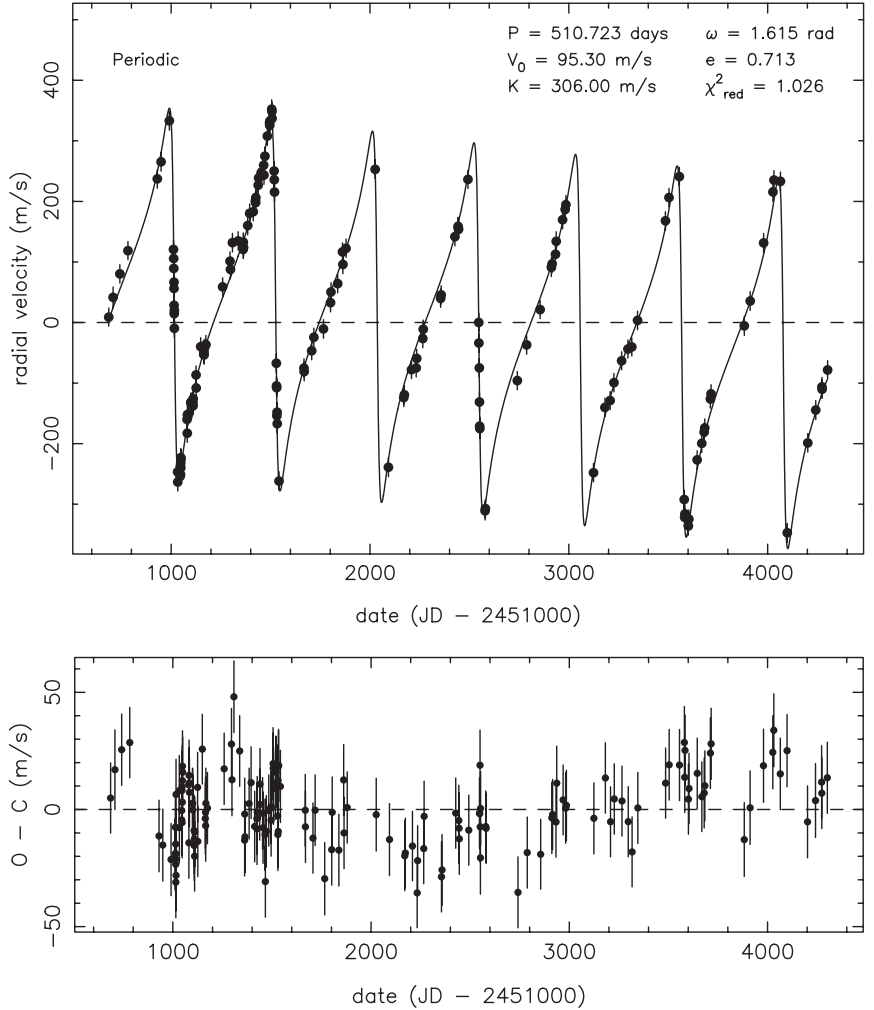

Figure 1. Radial velocity measurements of $\iota$ Dra along with the best-fit orbital solution (solid line). The lower panel shows the residuals of the fit, observed minus calculated $(O-C)$.

orbit. The revised solution by Zechmeister et al. (2008) is a significant improvement, mostly due to the large increase in time baseline leading to a much better-constrained period. Here we combine the Lick Observatory data published by Zechmeister et al. (2008) with further data covering almost two additional cycles of the orbit. The additional data were also acquired with the $0.6 \mathrm{~m}$ Coudé Auxillary Telescope and the Hamilton Echelle Spectrograph at the Lick Observatory with the same techniques described by Zechmeister et al. (2008).

The combined data are shown in Figure 1 along with the bestfit Keplerian model. A noise component of $14.5 \mathrm{~m} \mathrm{~s}^{-1}$ was added in quadrature to the derived uncertainties for each measurement in order to force $\chi^{2} \sim 1$. As was noted by Zechmeister et al. (2008), the addition of a linear trend of $\sim-13.65 \mathrm{~m} \mathrm{~s}^{-1} \mathrm{yr}^{-1}$ is required to provide an adequate fit to the data. The residuals to the fit, shown in the lower panel of Figure 1, have an rms of $15.05 \mathrm{~m} \mathrm{~s}^{-1}$. This level of stellar jitter is common for evolved stars (Wright 2005). Zechmeister et al. (2008) find a particularly strong stellar oscillation signature for $\iota$ Dra with a frequency of $3.8 \mathrm{day}^{-1}$. A Fourier analysis of the residuals did not reveal any further significant periodicity. The orbital parameters for the fit presented here are shown in Table 1, along with the parameters determined by Zechmeister et al. (2008) for comparison.

Although the eccentricity and periastron argument have comparable precisions resulting from this fit, the ephemeris critical parameters of period and time of periastron passage (Kane et al. 2009) are significantly improved. This is represented in Figure 2, which shows the reduced $\chi^{2}$ maps of parameter space for both the period (solid line) and the periastron argument (dashed line), in which one parameter is varied while holding all other parameters fixed. The large number of orbital phases

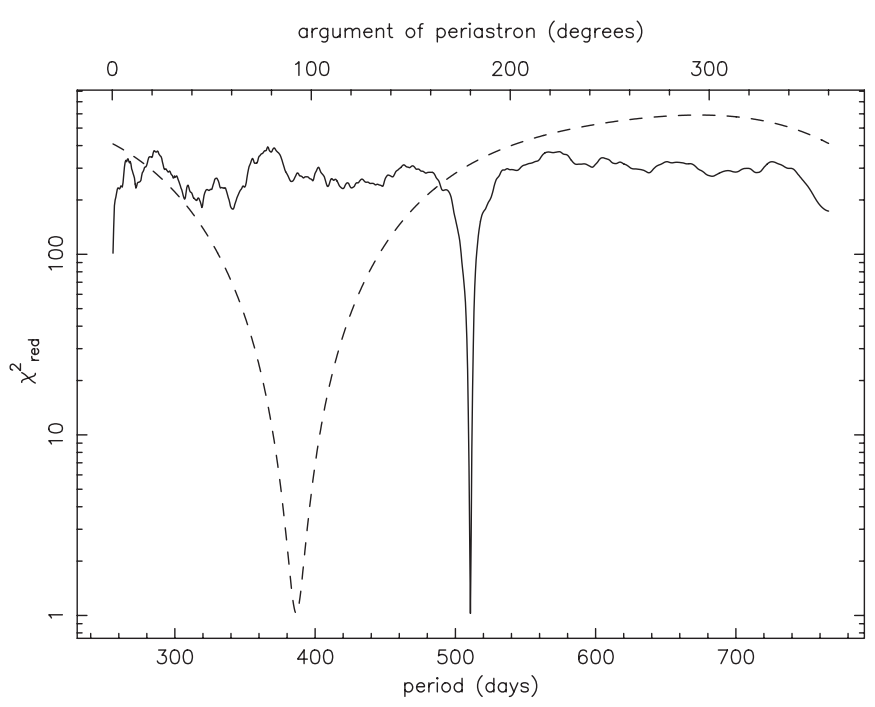

Figure 2. $\chi^{2}$ maps for the fitted period (solid line) and argument of periastron (dashed line).

Table 1

Fit Parameters for $\iota$ Dra

\begin{tabular}{lcc}
\hline \hline \multicolumn{1}{c}{ Parameter } & 2008 Fit & Revised Fit \\
\hline$P($ days $)$ & $510.88 \pm 0.15$ & $510.72 \pm 0.07$ \\
$K\left(\mathrm{~km} \mathrm{~s}^{-1}\right)$ & $299.9 \pm 4.3$ & $306.0 \pm 3.8$ \\
$\omega\left(^{\circ}\right)$ & $88.7 \pm 1.4$ & $92.5 \pm 0.7$ \\
$e$ & $0.7261 \pm 0.0061$ & $0.713 \pm 0.008$ \\
$t_{p}(\mathrm{JD}-2,450,000)$ & $2013.94 \pm 0.48$ & $2015.36 \pm 0.16$ \\
Linear trend $\left(\mathrm{m} \mathrm{s}^{-1} \mathrm{yr}^{-1}\right)$ & $-13.8 \pm 1.1$ & $-13.65 \pm 0.75$ \\
\hline
\end{tabular}

Note. The orbital parameters for $\iota$ Dra as measured by Zechmeister et al. (2008) and the revised orbital parameters based upon the combined data set are presented in this paper.

covered prevents significant global minima from occurring in period space, but the periastron argument is less constrained due to the need for more measurements during the eccentric (rapidly changing) phase of the planetary orbit.

\section{TRANSIT PROBABILITY, DEPTH, AND DURATION}

As described by Barnes (2007) and Kane \& von Braun (2008), the transit probability is a strong function of both the eccentricity and the argument of periastron. In particular, the transit probability is the strongest when the periastron passage occurs close to the star-observer line of sight, or where $\omega=90^{\circ}$. The orbit for $\iota$ Dra b, shown in Figure 3, is thus very well suited to produce a relatively high transit probability.

The other two primary factors that impact the transit probability are the radii of the planet and the host star. One can see from Figure 3 that $\iota$ Dra $b$ would have a similar transit probability to Mercury if it was not for these additional factors. To demonstrate the impact of the stellar radius and orbital eccentricity, Figure 4 shows how the transit probability varies as a function of eccentricity for three different luminosity classes for a K2 star. This uses the best-fit orbital parameters shown in Table 1 and assumes a planetary radius of one Jupiter radius. The effect of eccentricity is clearly dramatic for giant stars, driving the transit probability past $30 \%$ for eccentricities greater than $\sim 0.85$.

However, the dramatic positive effect the stellar radius has upon the transit probability results in an equally dramatic negative effect upon the transit depth. For the host star mass 


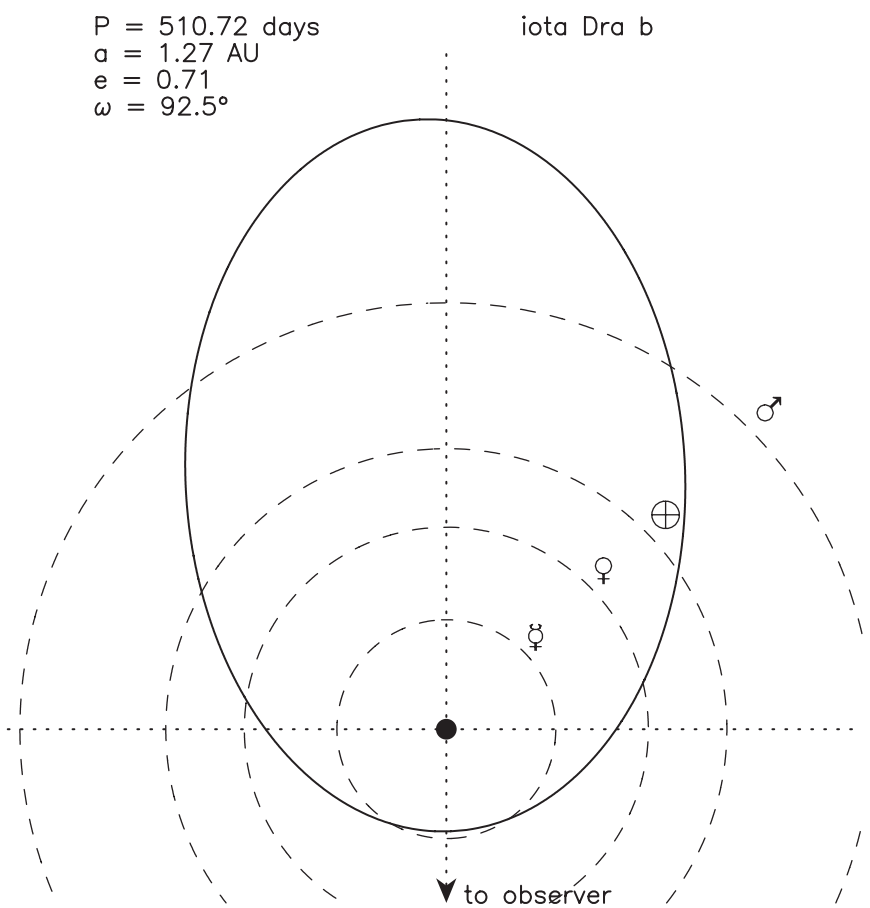

Figure 3. Orbit of the planet orbiting $\iota$ Dra (solid line) and the orbits of the solar system planets for comparison (dashed lines).

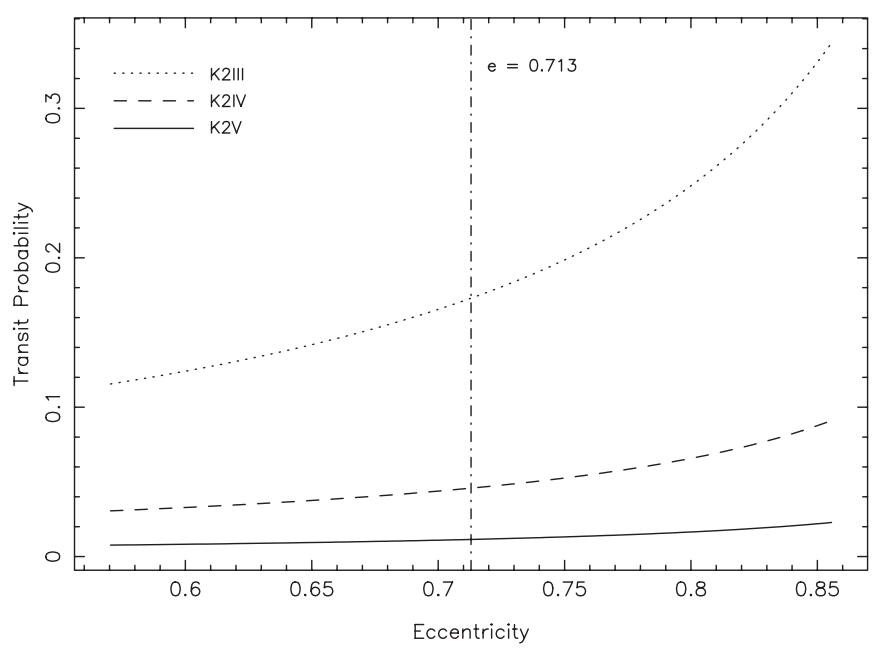

Figure 4. Transit probability as a function of eccentricity for three different stellar models showing the dramatic impact of the host being a giant star.

and radius, we adopt the values of $M_{\star}=1.05 M_{\odot}$ and $R_{\star}=12.88 R_{\odot}$, respectively, as measured by Allende Prieto \& Lambert (1999). This stellar mass produces a planetary mass of $M_{p} \sin i=8.7 M_{J}$ and a semimajor axis of $a=1.27 \mathrm{AU}$. Using the methods described in Bodenheimer et al. (2003), we estimate a planetary radius of $R_{p}=1.11 R_{J}$. The uncertainty in the stellar mass/radius and subsequent uncertainty in the planetary mass/ radius has a minor effect on the estimated transit duration but in no way effects the predicted transit midpoints since these are derived using the orbital parameters. Additionally, planetary radii become insensitive to mass in the high-mass regime, as demonstrated by the high-mass planets XO-3 b and CoRoT-3 b. Planetary radii have very little impact on both the transit probability and duration since $R_{p} \ll R_{\star}$, but the insensitivity of radius to mass as described makes this impact negligible in nature.
Table 2

Transit Probabilities, Depths, and Durations

\begin{tabular}{ccccc}
\hline \hline$R_{\star}$ & $e$ & $P_{t}(\%)$ & $\Delta F / F_{0}$ & $t_{d}$ (days) \\
\hline 1.00 & 0.000 & 0.41 & $1.30 \times 10^{-2}$ & 0.63 \\
1.00 & 0.713 & 1.42 & $1.30 \times 10^{-2}$ & 0.26 \\
12.88 & 0.000 & 4.74 & $7.84 \times 10^{-5}$ & 7.42 \\
12.88 & 0.713 & 16.52 & $7.84 \times 10^{-5}$ & 3.04 \\
\hline
\end{tabular}

Note. These calculations assume a planetary radius of $R_{p}=1.11 R_{J}$.

Table 2 shows the calculated transit probability $\left(P_{t}\right)$, depth, and duration $\left(t_{d}\right)$ for $\iota$ Dra $\mathrm{b}$ using the orbital parameters from Table 1. For comparative purposes, these parameters are also shown for a solar radius star. The problem of the transit depth becomes very apparent when one draws this comparison and, as described earlier, is one of the main hindrances to groundbased detection of such transits. The expected long duration of the transit is also problematic since ground-based coverage of a transit from a single telescope is impossible. These issues are described in more detail in the following sections.

\section{TRANSIT EPHEMERIS REFINEMENT}

The accuracy of the predicted transit midpoint and the size of the time window in which the complete transit could occur are of course highly dependent upon the uncertainties associated with the orbital parameters. The calculations for predicting transit ephemerides and the influence of period and time of periastron passage are described in detail by Kane et al. (2009). Based upon the improved orbital parameters shown in Table 1, the transit ephemeris for $\iota$ Dra $b$ has been calculated for the next 10 years and is shown in Table 3.

The size of the new predicted transit window is 73.56 days based upon the orbital parameters of Frink et al. (2002) and 6.05 days based upon the orbital parameters of Zechmeister et al. (2008). With the improved orbital parameters described in this paper, the next predicted transit window has been reduced in size to 4.35 days. By the time the 2020 transit prediction arrives, the size of the transit window will have grown to 5.33 days. It should be noted that, despite the remaining large size of the transit window, it is now largely dominated by the transit duration, which is expected to be $\sim 3.0$ days as shown in Table 2. Even so, attempts to obtain full coverage of the transit window from the ground will require a multi-longitudinal campaign during which one can only hope for cooperative weather. The complete observation of an ingress or egress during a single night is a substantially more achievable goal under such circumstances. However, one still must contend with the challenge of meeting the photometric precision requirements for a successful detection.

\section{PHOTOMETRIC STABILITY}

SIMBAD refers to $\iota$ Dra as a variable star based on its citation as NSV 7077 in the New Catalogue of Suspected Variable Stars (Kukarkin et al. 1982). The NSV entry is based on the photometric study of Jackisch (1963), who reported a magnitude range of 0.09 mag. Later, Percy (1993) included $\iota$ Dra in his search for photometric variability in $\mathrm{K}$ giants chosen from the Bright Star Catalogue (Hoffleit \& Jaschek 1991) and found the star to be constant to a limit of $0.01 \mathrm{mag}$.

We investigated the photometric stability of $\iota$ Dra using newer observations. The Hipparcos satellite observed the star during its three-year mission and acquired a photometric data 
Table 3

Refined Transit Ephemeris for $\iota$ Dra b

\begin{tabular}{|c|c|c|c|c|c|}
\hline \multicolumn{2}{|c|}{ Beginning } & \multicolumn{2}{|c|}{ Midpoint } & \multicolumn{2}{|c|}{ End } \\
\hline JD & Date & JD & Date & JD & Date \\
\hline 2455587.81 & 201101260720 & 2455589.98 & 201101281135 & 2455592.16 & 201101301550 \\
\hline 2456098.46 & 201206192256 & 2456100.70 & 201206220452 & 2456102.95 & 201206241048 \\
\hline 2456609.11 & 201311121432 & 2456611.42 & 201311142209 & 2456613.74 & 201311170546 \\
\hline 2457119.76 & 201504070608 & 2457122.14 & 201504091526 & 2457124.53 & 201504120043 \\
\hline 2457630.41 & 201608292144 & 2457632.86 & 201609010843 & 2457635.32 & 201609031941 \\
\hline 2458141.06 & 201801221320 & 2458143.58 & 201801250159 & 2458146.11 & 201801271438 \\
\hline 2458651.71 & 201906170456 & 2458654.30 & 201906191916 & 2458656.90 & 201906220936 \\
\hline 2459162.36 & 202011082032 & 2459165.02 & 202011111233 & 2459167.69 & 202011140434 \\
\hline
\end{tabular}

Notes. The columns indicate the beginning, midpoint, and end of the transit window in both Julian and calendar dates for the next 10 years. The calendar date is expressed in UT and includes the year, month, day, hour, and minute.

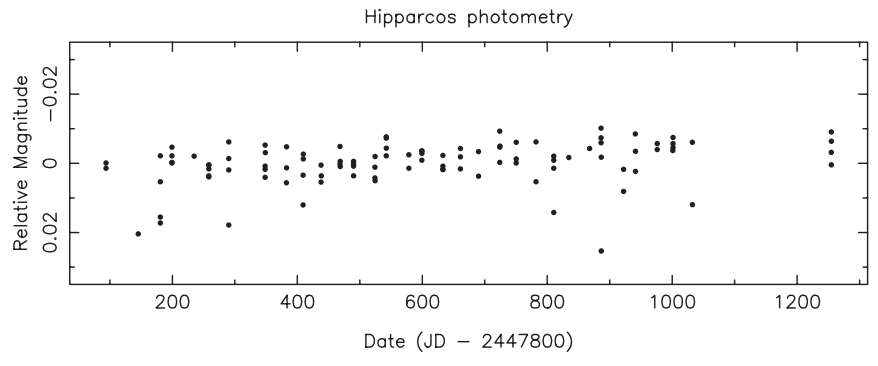

Figure 5. Photometry of $\iota$ Dra from the Hipparcos mission.

set consisting of 104 measurements spanning a period of 1160 days (Perryman et al. 1997). The scatter of the 104 $\iota$ Dra measurements is $0.005 \mathrm{mag}$, while the range of the observations, defined in terms of the 5th and 95th percentiles of their distribution, is $0.02 \mathrm{mag}$. The scatter is roughly consistent with the expected uncertainty of a single observation, but the range is roughly twice that expected from a constant star. Consequently, the Hipparcos Catalogue (Perryman et al. 1997) lists the variability type for $\iota$ Dra as a blank, indicating that the star "could not be classified as variable or constant." We performed a Fourier analysis of the Hipparcos data, plotted in Figure 5, and confirmed the absence of any significant periodic variability.

We also acquired new Johnson $B$ and $V$ observations with the T3 $0.4 \mathrm{~m}$ Automatic Photoelectric Telescope (APT) located at Fairborn Observatory in the Patagonia Mountains of southern Arizona. Between 2010 January and May, T3 observed $\iota$ Dra differentially with respect to a nearby comparison star in the following sequence, termed a group observation: $S, C, V, C, V, C, V, C, S$, where $S$ is a sky reading, $C$ is the comparison star HD $144284=$ $\theta$ Dra $(V=4.01, B-V=0.53, \mathrm{~F} 8 \mathrm{IV})$, and $V$ is the program (variable?) star $\iota$ Dra $(V=3.29, B-V=1.17$, K2 III). A 2.3 mag neutral-density filter was used in combination with the $B$ and $V$ filters to attenuate the signal and so minimize the dead-time correction for the two bright stars. Three $V-C$ differential magnitudes in both $B$ and $V$ were computed from each sequence and averaged to create $B$ and $V$ group means. Group mean differential magnitudes with internal standard deviations greater than $0.01 \mathrm{mag}$ were rejected to eliminate observations taken under non-photometric conditions. The surviving group means were corrected for differential extinction with nightly extinction coefficients, transformed to the Johnson system with yearly mean transformation coefficients, and treated as single observations thereafter. The typical precision of a single group-
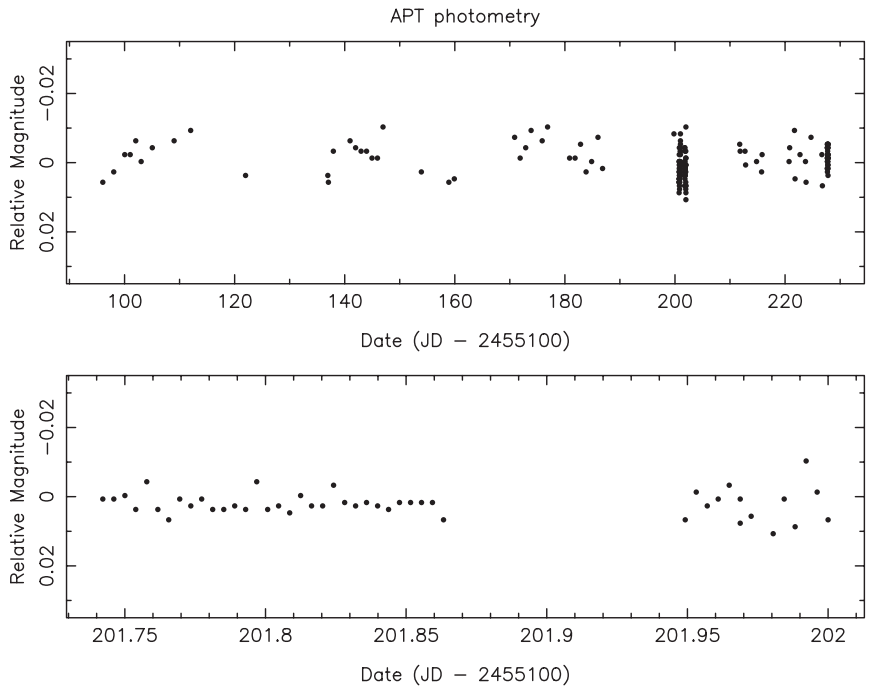

Figure 6. Johnson $B$ photometry of $\iota$ Dra obtained using the T3 $0.4 \mathrm{~m} \mathrm{APT} \mathrm{at}$ the Fairborn Observatory showing long-term (top panel) and short-term (bottom panel) stability of $\sim 0.004$.

mean observation from $\mathrm{T} 3$, as measured for pairs of constant stars, is $\sim 0.004-0.005$ mag (Henry et al. 2000). The APT acquired one or two group observations each clear night except for three full nights when the star was observed at a much higher cadence of $\sim 10$ group observations per hour. The APT collected a total of $224 B$ and $220 \mathrm{~V}$ group observations. Further details on the automatic operation of this telescope, the observing procedures, and the data reduction process can be found in Henry et al. (2000) and references therein.

The complete, reduced Johnson $B$ data set is plotted in the top panel of Figure 6; the bottom panel presents just the highcadence $B$ photometry from one of the three monitoring nights. The data in both panels scatter about their means with a standard deviation of $0.0041 \mathrm{mag}$, after a half-dozen outliers are removed in each case. Results for the $V$ observations are essentially identical $(0.0043 \mathrm{mag})$. Thus, our observations suggest that $\iota$ Dra, as well as its comparison star $\theta$ Dra, are both constant to a limit of approximately $0.004 \mathrm{mag}$.

We note, however, that the first half of the complete $B$ and $V$ light curves suggest possible very low amplitude variability on a timescale of $\sim 30$ days. The variation then seems to damp out during the second half of the light curve. We performed Fourier analyses of the complete $B$ and $V$ data sets and found 


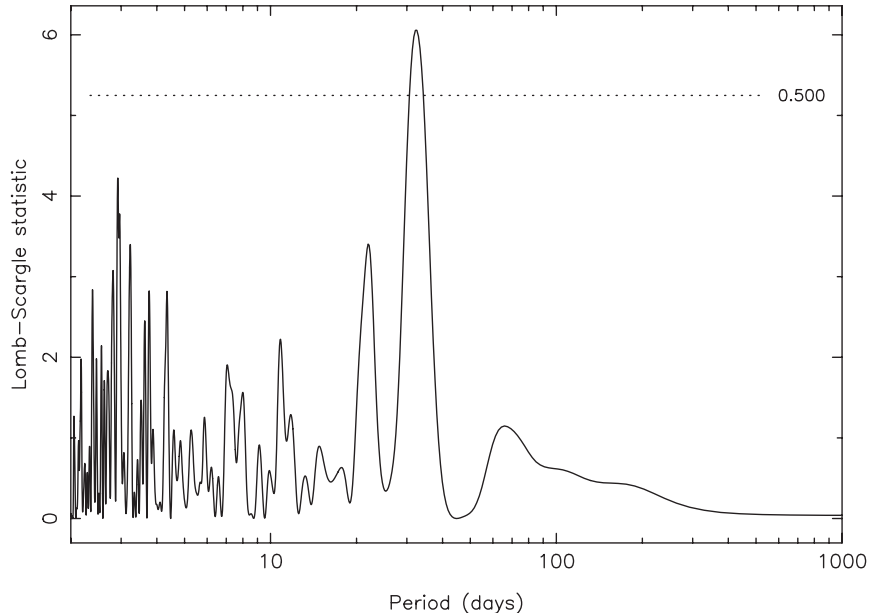

Figure 7. Periodogram of the $B$ observations acquired with the T3 APT. No strong periodicity is seen, but the highest peak at 32 days is consistent with the inspection of the first half of the light curve.

no significant periodic variability in either case (see Figure 7 for the $B$ data). These results are somewhat reminiscent of the light curves and period analyses of the $\mathrm{K}$ giants presented in the survey of Henry et al. (2000). Further observations will be required to determine if the apparent low-amplitude variability is real. For now, we claim only that $\iota$ Dra is constant to $\sim 0.004$ mag. We also conclude that the photometric variability reported by Jackisch (1963) is spurious and that $\iota$ Dra has yet to prove its status as a variable star.

In addition to improving the radial velocity orbit of $\iota$ Dra $b$ and detecting a linear trend in the residuals, Zechmeister et al. (2008) also found evidence for short-period radial velocity variations in the range $0.25-0.33$ days (we note that corresponding shortperiod photometric variations are not seen in our three nights of high-cadence observations). Using the radius measured by Allende Prieto \& Lambert (1999) of $R_{\star}=12.88 R_{\odot}$ and the rotational velocity measured by de Medeiros \& Mayor (1999) of $1.5 \mathrm{~km} \mathrm{~s}^{-1}$, we estimate the expected rotation period of $\iota$ Dra to be $\sim 434$ days. Thus, the possible low-amplitude variability seen in our photometry and the short-period, low-amplitude radial velocity variability found by Zechmeister et al. (2008) cannot be caused by the rotation of the star. Zechmeister et al. (2008) note that their short-period radial velocity variations may be similar to solar-type oscillations. Likewise, Henry et al. (2000) concluded that the low-amplitude, short-period photometric variability found in their sample of $\mathrm{K}$ giants could not be due to rotational modulation but had timescales consistent with radial pulsation.

\section{PROSPECTS FOR TRANSIT DETECTION}

\subsection{Photometric Detection}

The prospects for successful detection of a transit of $\iota$ Dra $b$ rely upon achieving the necessary photometric precision. The brightness of the star makes it a difficult target to observe with traditional CCD photometry due to saturation. Groundbased observations with photoelectric photometers, such as the APTs, mitigate this problem but are still challenged by the $<0.0001$ mag transit depth. Assef et al. (2009) suggest observing the star during the transit window using narrowband filters which would isolate the $\mathrm{Ca}$ II $\mathrm{H}$ and $\mathrm{K}$ chromospheric emission at the stellar limb, the effect of which will be to increase the depth of the transit.
While we did not definitely detect photometric variability in $\iota$ Dra, the amplitude and timescale of possible photometric variations are significantly larger than the predicted transit duration. Hence, two possibilities present themselves for detection of the transit in the face of these potential variations. If the periodic variation is well defined over long time periods, then it could be accurately modeled and subtracted from the data. Regardless, the transit window is shorter such that the variation is not expected to impact the transit detection on those timescales. Although the long transit duration prevents the monitoring of a complete transit within a single night, groundbased observations of a complete transit window of HD 80606b have been accomplished by Hidas et al. (2010) by obtaining multi-longitudinal coverage. In addition, the required cadence is much lower than for a typical hot Jupiter which allows greater flexibility in binning the measurements for greater precision.

Another possibility is to attempt complete monitoring of the transit window from a space-based observatory. Consider the case of HD 209458b for which the host star is a $V=7.65$ mainsequence star. Transits of this planet have been observed by both the Hubble Space Telescope (HST) and the Microvariability and Oscillations of Stars (MOST) satellite at very high precision. The HST observations by Brown et al. (2001) achieved a precision of $1.1 \times 10^{-4}$ and the MOST observations by Croll et al. (2007) achieved a precision of $3.5 \times 10^{-3}$. Assuming a precision which is dominated by photon noise, these values convert to $\iota$ Dra precisions of $1.5 \times 10^{-5}$ and $4.7 \times 10^{-4}$ for $H S T$ and MOST, respectively. These predicted precisions bring the detection of a transit of $\iota$ Dra $b$ within reach since the necessarily high cadence of these observations will require binning of the data over the long transit duration. It should be noted however that observations of $\iota$ Dra with MOST are difficult because it is significantly north of the Continuous Viewing Zone (CVZ).

\subsection{Spectroscopic Detection}

The Rossiter-McLaughlin (RM) effect (McLaughlin 1924; Rossiter 1924) provides a means whereby an exoplanet may be detected and indeed discovered spectroscopically. The first precedent for discovering a planet through this method occurred with the well-known planet orbiting HD 189733 (Bouchy et al. 2005). The application of the RM effect to transiting exoplanets has been described in detail by Gaudi \& Winn (2007). The amplitude of the RM effect, $K_{R}$, is

$$
K_{R} \equiv V_{S} \sin I_{S} \frac{\gamma^{2}}{1-\gamma^{2}},
$$

where $V_{S} \sin I_{S}$ is the rotational velocity of the star and $\gamma$ is the transit depth.

The proportionality of the RM amplitude with the rotational velocity of the star presents an immediate large bias against giant stars due to their relatively slow rotation rate. For $\iota$ Dra, the rotational velocity has been measured by de Medeiros \& Mayor (1999) to be $1.5 \mathrm{~km} \mathrm{~s}^{-1}$. Using the estimated transit depth of $7.84 \times 10^{-5}$ from Table 2 , the amplitude of the RM effect as the planet transits is predicted to be only $12 \mathrm{~cm} \mathrm{~s}^{-1}$. Any attempt to measure such a spectroscopic transit signature will undoubtedly require awaiting the next generation of radial velocity instruments.

\section{CONCLUSIONS}

Inadequate precision of orbital parameters presents a major hindrance to testing the transit hypothesis of planets 
discovered using the radial velocity technique. The challenge of improving transit ephemerides and monitoring transit windows is currently being met by such groups as the TERMS. The value of such a survey includes an improved understanding of planetary orbits and the potential for gaining insight into the exoplanet mass-radius relationship for the brightest host stars.

For giant stars, this regime is largely unexplored even though giant stars have the highest transit probabilities since $P_{t} \propto R_{\star}$. Eccentric orbits of planets around giant stars are relatively rare, with only a handful exceeding an eccentricity of 0.5 , including HD 122430b and $\iota$ Dra b. The planet orbiting HD 13189 (Hatzes et al. 2005) has an eccentricity of 0.28 but this is more than compensated for by the host; a K2 II star with an estimated radius exceeding $50 R_{\odot}$.

The case of $\iota$ Dra b is particularly interesting since the stellar radius and orbital elements create a transit probability exceeding that of a hot Jupiter $(P<5$ days $)$, despite having an orbital period of more than 500 days. Our refined orbital solution produces a transit window that is accessible to a concerted ground-based effort or modest space-based effort with potentially high return for a successful transit detection. In addition, our photometry shows the short-term stability of $\iota$ Dra to be comparatively favorable for typical late-type giant stars. A Fourier analysis of the photometry reveals a periodic signal at $\sim 32$ days, although the weakness of the signal makes its presence inconclusive and we conclude that $\iota$ Dra is constant at the $\sim 0.004$ mag level. However, the challenge of achieving the needed photometric precision required to achieve an unambiguous detection for the transit will likely require spaced-based observations during the transit window.

The authors thank David Ciardi and Andrew Howard for several useful suggestions. We thank the many CAT observers who have observed $\iota$ Dra over the years, especially David Mitchell and Saskia Hekker. Contributions of Andreas Quirrenbach to the $\mathrm{K}$ giants radial velocity monitoring program at the Lick Observatory are gratefully acknowledged. This research has made use of the NASA/IPAC/NExScI Star and Exoplanet Database, which is operated by the Jet Propulsion Laboratory, California Institute of Technology, under contract with the National Aeronautics and Space Administration.

\section{REFERENCES}

Allende Prieto, C., \& Lambert, D. L. 1999, A\&A, 352, 555

Assef, R. J., Gaudi, B. S., \& Stanek, K. Z. 2009, ApJ, 701, 1616

Barbieri, M., et al. 2007, A\&A, 476, L13

Barnes, J. W. 2007, PASP, 119, 986

Batygin, K., Bodenheimer, P., \& Laughlin, G. 2009, ApJ, 704, L49

Bodenheimer, P., Laughlin, G., \& Lin, D. N. C. 2003, ApJ, 592, 555

Bouchy, F., et al. 2005, A\&A, 444, L15

Brown, T. M., Charbonneau, D., Gilliland, R. L., Noyes, R. W., \& Burrows, A. 2001, ApJ, 552, 699

Croll, B., et al. 2007, ApJ, 658, 1328

da Silva, L., et al. 2006, A\&A, 458, 609

de Medeiros, J. R., \& Mayor, M. 1999, A\&AS, 139, 433

Döllinger, M. P., Hatzes, A. P., Pasquini, L., Guenther, E. W., \& Hartmann, M. 2009, A\&A, 505, 1311

Frink, S., Mitchell, D. S., Quirrenbach, A., Fischer, D. A., Marcy, G. W., \& Butler, R. P. 2002, ApJ, 576, 478

Gaudi, B. S., \& Winn, J. N. 2007, ApJ, 655, 550

Hatzes, A. P., Guenther, E. W., Endl, M., Cochran, W. D., Döllinger, M. P., \& Bedalov, A. 2005, A\&A, 437, 743

Henry, G. W., Fekel, F. C., Henry, S. M., \& Hall, D. S. 2000, ApJS, 130, 201

Hidas, M. G., et al. 2010, MNRAS, 406, 1146

Hoffleit, D., \& Jaschek, C. V. 1991, The Bright Star Catalogue (5th ed.; New Haven, CT: Yale Univ. Obs.)

Ida, S., \& Lin, D. N. C. 2005, ApJ, 626, 1045

Jackisch, G. 1963, in Veroeffentlichungen der Sternwarte in Sonneberg (Publications of Sonneberg Observatory), Bd. 5, Heft 5 (Berlin: Academic), 232

Kane, S. R., Mahadevan, S., von Braun, K., Laughlin, G., \& Ciardi, D. R. 2009, PASP, 121, 1386

Kane, S. R., \& von Braun, K. 2008, ApJ, 689, 492

Kane, S. R., \& von Braun, K. 2009, PASP, 121, 1096

Kennedy, G. M., \& Kenyon, S. J. 2008, ApJ, 673, 502

Kukarkin, B. V., et al. 1982, New Catalogue of Suspected Variable Stars (Moscow: Academy of Sciences)

Laughlin, G., Deming, D., Langton, J., Kasen, D., Vogt, S., Butler, P., Rivera, E., \& Meschiari, S. 2009, Nature, 457, 562

McLaughlin, P. R. 1924, ApJ, 60, 22

Moutou, C., et al. 2009, A\&A, 498, L5

Niedzielski, A., Goździewski, K., Wolszczan, A., Konacki, M., Nowak, G., \& Zieliński, P. 2009, ApJ, 693, 276

Percy, J. R. 1993, PASP, 105, 1422

Perryman, M. A. C., et al. 1997, A\&A, 323, L49

Rossiter, R. A. 1924, ApJ, 60, 15

Sadakane, K., Ohnishi, T., Ohkubo, M., \& Takeda, Y. 2005, PASJ, 57, 127

Setiawan, J., Pasquini, L., da Silva, L., Hatzes, A. P., von der Lühe, O., Girardi, L., de Medeiros, J. R., \& Guenther, E. 2004, A\&A, 421, 241

Veras, D., Crepp, J. R., \& Ford, E. B. 2009, ApJ, 696, 1600

Wright, J. T. 2005, PASP, 117, 657

Zechmeister, M., Reffert, S., Hatzes, A. P., Endl, M., \& Quirrenbach, A. 2008, A\&A, 491, 531 